ORIGINAL

\title{
GRADO DE CUMPLIMIENTO DE LA LEGISLACIÓN E IMPARTICIÓN DE ENSEÑANZAS ANTI-TABACO EN LOS CENTROS ESCOLARES ESPAÑOLES
} \author{
Miguel Torrecilla (4), Mar González Bustos (5) y Mª Dolores Plaza (4) \\ (1) Unidad Docente de Medicina Familiar y Comunitaria de Salamanca \\ (2) Servicio de Neumología, Hospital Universitario de Salamanca \\ (3) Servicio de Neumología, Hospital de la Princesa de Madrid \\ (4) Centro de Salud San Juan (Salamanca) \\ (5) Servicio de Radioterapia, Hospital Universitario de Salamanca
}

Miguel Angel Hernández-Mezquita (1), Miguel Barrueco (2), Carlos Andrés Jiménez-Ruiz (3),

\section{RESUMEN}

Fundamento: No se dispone de estudios que analicen el cumplimiento de la legislación antitabaco en los centros escolares españoles, por lo que nos propusimos conocer el grado de cumplimiento de la legislación oficial anti-tabaco en dichos centros y la influencia que ello tiene sobre el porcentaje de alumnos fumadores.

Método: Se realizó una encuesta entre 3.050 directores de centros educativos españoles, a los que se les preguntó sobre điversos aspectos del cumplimiento de la legislación oficial antitabaco en los centros y sobre las principales características del consumo de tabaco en los mismos.

Resultados: El 80,9\% de los directores afirman que en sus centros se exige el cumplimiento de la legislación y el $64,9 \%$ disponen de carteles que advierten de la prohibición de fumar. Afirman impartir enseñanzas anti-tabaco el $82,2 \%$ de los centros en los que se exige la legislación y en el $64,8 \%$ de los centros en los que esta legislación no se exige. Creen que el porcentaje de profesores que fuman ante los alumnos es del $5,9 \%$ en los centros en que se exige la legislación y del $12,9 \%$ en los que no se cumple. En los centros donde se imparten enseñanzas anti-tabaco creen que han probado el tabaco el 29\% de los alumnos, frente al $31 \%$ que creen lo han hecho en los centros donde no se imparten estas enseñanzas $(p<0,001)$.

Conclusiones: El cumplimiento de la legislación es insuficiente. Los directores de los centros educativos, como encargados de velar por el cumplimiento de la legislación oficial anti-tabaco en sus centros. deben exigir el cumplimiento de esta legislación, ya que esta medida es eficaz para mejorar el funcionamiento global de los centros y para reducir los porcentajes de alumnos y profesores fumadores. fesores.

Palabras clave: Tabaco. Salud escolar. Legislación. Pro-

\section{ABSTRACT}

\section{Anti-tobacco laws compliance and health education in the spanish schools}

Objective: knowing the dregree of fulfilment of the anti tobacco legislation in Spanish schools and the influence that this has on the percentage of smoking pupils.

Material and methods: a survey was carried out among 3050 headmasters of Spanish schools, who were asked about different aspects of the fulfilment of the antitobacco laws in their schools and about the main characteristics of tobacco consumption in their centers.

Results: a $80,9 \%$ of the headmasters claim that the fulfitment of the legislation is demanded in their centers, and only a $64.9 \%$ of them have posters in theirs schools that warn people albout smoking ban. Anti tobacco teachings are imparted in a $82.2 \%$ of the schools where laws is demanded and only in a $64.8 \%$ where is not demanded. The percentage of teachers who, smoke in the presence of the pupils is of a 5.9\% in the schools where anti tobacco legislation is demanded and a $12.9 \%$ where is not demanded. In the schools where anti tobacco teachings are imparted, a $29 \%$ of the pupils have tried tobacco, opposite a $31 \%$ who have tried in the schools where these teachings are not imparted.

Conclusions: the, headmasters of the schools, as people in charge of watching over the fulfilment of the anti tobacco legislation in theirs schools, must demand the fulfilment of this one, because this measure is effective in order to improve the global functioning of the schools and to reduce the percentages of smoking pupils and teachers.

Key words: tobacco, school, laws, teachers.
Correspondencia:

Miguel Barrueco Ferrero

Servicio de Neumología Hospital Universitario de Salamanca.

Po San Vicente 58-182.

37007 Salamanca

Telf.: 923291306

Correo electrónico: mibafe@gugu.usal.es

\section{INTRODUCCIÓN}

En nuestro medio, el inicio al consumo de tabaco se produce en la edad escolar. La escuela es uno de los lugares donde los niños comienzan a fumar por varias razones, de las 
que la principal es que la iniciación es, en general, un proceso de grupo. Fumar a estas edades refuerza la incorporación al grupo y fortalece las interrelaciones dentro del mismol. Casi el $90 \%$ de los jóvenes manifiestan haber fumado su primer cigarrillo en compañía de otros jóvenes, frente al 10\% que manifiestan haber fumado su primer cigarrillo en solitario $^{2}$. Otro factor directamente relacionado con la iniciación en el consumo de tabaco de los jóvenes es la percepción que éstos tienen del número de adultos y compañeros que fuman. A medida que el porcentaje de compañeros fumadores aumenta, disminuye el sentimiento de culpabilidad por fumar y aumenta el porcentaje de fumadores, ya que hace que este hábito sea considerado como "normal", al funcionar como mecanismo de feed-back positivo" ${ }^{2}$ Particular atención merece el papel que el profesor juega a los ojos de los alumnos y la coherencia que trasmite a través de su propia conducta personal. Este es el denominado "papel ejemplarizante", que hace que en ocasiones el denominado "currículum oculto" enmascare el "educativamente correcto" mensaje teórico.

El actual marco legislativo español intenta proteger a los alumnos de las actitudes poco ejemplarizantes de algunos profesores, prohibiéndoles a éstos fumar en las áreas de convivencia con los alumnos, pero el cumplimiento de estas normas es variable, probablemente debido a que tanto los profesores fumadores como los responsables de hacer cumplirlas, aún no están suficientemente informados o motivados. Por otra parte, en muchas escuelas no están señalizadas las áreas donde no se permite fumar ni existen carteles recordando la legislación vigente. Por todo ello, son necesarias medidas dirigidas a conseguir que las escuelas jueguen un papel más determinante en el fomento del no fumar ${ }^{3}$.

Los países occidentales han desarrollado en los últimos años una variada legislación anti-tabaco con el objetivo de proteger a los ciudadanos, especialmente a los menores y a quienes no desean verse expuestos al humo de tabaco ${ }^{4}$. En España la legislación específi- ca más importante acerca del tabaco es la contenida en el Real Decreto 192/885. Este Real Decreto establece las limitaciones en la venta y uso de tabaco a menores y la prohibición de fumar en los centros escolares.

La Administración también contempla la prevención del tabaquismo dentro de los contenidos de la Educación para la Salud (EpS) como una materia fundamental integrada en el currículum cscolar, con carácter de disciplina transversal. En la actualidad no se conoce el número de colegios que imparten $\mathrm{EpS}$ ni cuántos imparten temas anti-tabaco dentro de la $\mathrm{EpS}^{4}$.

El marco legislativo español ${ }^{6-11}$, así como las Ordenes de las Consejerías de Educación de las distintas comunidades autónomas con competencias en materia de educación, conforman un marco adecuado que permite a los centros educativos fomentar y desarrollar los programas educativos anti-tabaco.

Por todo lo expuesto, el principal objetivo de nuestro estudio ha sido conocer el grado de cumplimiento de la legislación antitabaco en los centros educativos del estado español y la influencia que este grado de cumplimicnto cjerce sobre los contenidos destinados a la prevención del tabaquismo que los jóvenes reciben durante el tiempo que asisten a los centros educativos y sobre la conducta ante el tabaco de los alumnos.

\section{SUJETOS Y MÉTODOS}

El estudio fuc dirigido a directores escolares de los centros educativos que impartían enseñanza primaria, secundaria o ambas conjuntamente en España durante el curso académico 1996-199712. Se eligió a los directores escolares por ser profesionales altamente cualificados, con muchos años de experiencia en el trato con jóvenes y por entender que, por el papel que tienen en la comunidad escolar (representantes de la administración, miembros del claustro y profesores del centro), disponen de información privilegiada acerca del mundo escolar, además de ser los encar- 
gados de velar por el cumplimiento de la legislación oficial anti-tabaco en los centros educativos.

Se diseñó una encuesta preparada para ser autocumplimentada de forma anónima y para ser corregida de forma automática por el Centro de Proceso de Datos de la Universidad de Salamanca (CPDUS). La encuesta incluía preguntas referentes a las características de los centros (tipo de educación impartida y titularidad), a la conducta de fumar en los centros (porcentajes de alumnos y profesores fumadores), a la exigencia o no del cumplimiento de la legislación (presencia o no de carteles advirtiendo de la prohibición de fumar) y a los contenidos educativos de EpS y relativos a la prevención del tabaquismo que se imparten en los centros educativos españoles. En el
Anexo 1 se exponen las principales preguntas incluidas en la encuesta.

La encuesta se envió a 8.000 directores, elegidos al azar, del total de los 18.457 existentes. A todos ellos se les adjuntó un sobre prefranqueado para facilitar la respuesta. De las 8.000 encuestas enviadas $(43,3 \%$ del total de centros educativos del estado español en el curso académico 1996-97), se recibió la respuesta de 3.156, de las que, tras retirar aquellas encuestas cumplimentadas defectuosamente, finalmente resultaron válidas para el estudio las respuestas de 3.050 centros educativos ( $16,52 \%$ del total), que forman la muestra objeto de análisis de este trabajo de investigación ${ }^{13}$. El estudio, presenta una desviación máxima del $\pm 1,65 \%$ para resultados poblacionales, con un nivel de confianza del $95,5 \%$. El proceso se recoge de forma gráfica en la figura 1.

Figura 1

Proceso de selección de la muestra objeto de estudio

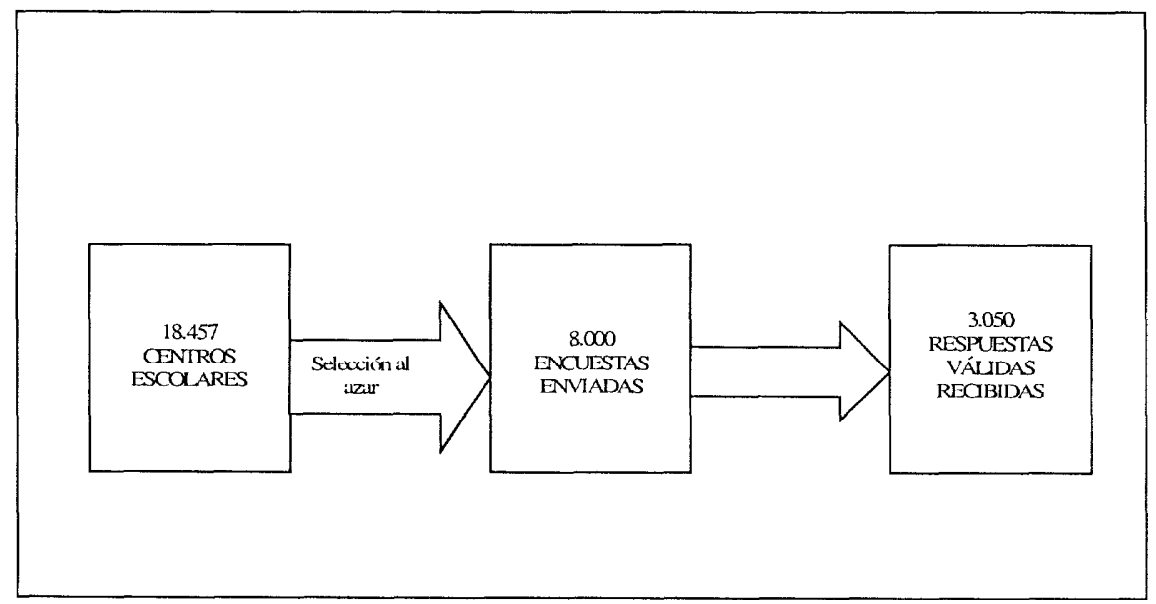

Los datos fueron procesados por el CPDUS, donde fueron codificados y posteriormente tratados con el programa estadístico SPSS 6.1.2 para Windows. Se empleó el $T$ test para comparación de medias cuando la variable dependiente era cuantitativa y la independiente cualitativa de dos categorías, chi $^{2}$ para variables categóricas cuando las dos variables eran cualitativas, Anova de 1 factor (análisis de varianza) cuando la variable dependiente era cuantitativa y la independiente cualitativa de más de dos categorías (en este caso, si se encontraban diferencias significativas se aplicó la prueba de Scheffe a posteriori, para ver cntre qué categorías las diferencias eran significativas) y regresión múltiple 
cuando la variable dependiente era cuantitativa y las variables predictoras cualitativas.

\section{RESULTADOS}

Un total de 2.465 directores $(80,9 \%)$ afirman que en sus centros se exige por norma el cumplimiento de la legislación oficial antitabaco y $585(19,1 \%)$ reconocen que no exigen el cumplimiento dicha legislación. Existen carteles recordando la prohibición de fumar en 1.980 centros $(64,9 \%)$ y no existen en $1.070(35,1 \%)$. En aquellos centros cuyos directores declaran que exigen el cumplimiento de la legislación oficial anti-tabaco, el porcentaje de centros en los que existen carteles que informan de la prohibición de fumar es mayor que en los centros en los que no se exige dicha legislación $(68,9 \%$ y $51,4 \%$ respectivamente; $p<0,001)$. En la figura 2 se representan los porcentajes de centros que cumplen la legislación y que cuentan con carteles anti-tabaco.

\section{Figura 2}

Porcentajes de centros que los directores afirman que cumplen la legislación y de centros que cuentan con carteles que informan de la prohibición de fumar en el centro



Se imparten contenidos de EpS en 2.655 centros $(87 \%)$ y se imparten temas anti-tabaco dentro de la EpS, en 2.317 centros (76\% del total), mientras que en $733(24 \%)$ no se imparten. En los centros donde se exige el cumplimiento de la legislación, se imparten temas anti-tabaco en el $82,2 \%$ de los casos, mientras que en los centros donde no se exige, el porcentaje de centros que imparten temas anti-tabaco es significativamente más bajo $(64,8 \%$, p $0<, 001)$.

No hay diferencias en el porcentaje medio estimado de profesores fumadores entre 
los centros que exigen el cumplimiento de la lcgislación y los que no lo exigen $(29,25 \pm$ 18,2 frente a $32,97 \pm 18,8 ; p>0,05$ ). En cambio, el porcentaje medio estimado de profesores que fuman ante los alumnos en los centros en que se exige el cumplimiento de la legislación es del $5,9 \pm 12,9$, mientras que en los centros en que no se exige es del $12,9 \pm$ $20,3(p<0,001)$. En la figura 3 se representan los porcentajes de centros que imparten enseñanzas anti-tabaco y de profesores que se cstima fuman ante los alumnos en función de la exigencia del cumplimiento de la legislación en los centros.

Figura 3

Porcentajes de centros en los que se imparten enseñanzas anti-tabaco y de profesores que fuman ante los alumnos en los centros que afirman cumplir la legislación y en los que no la cumplen

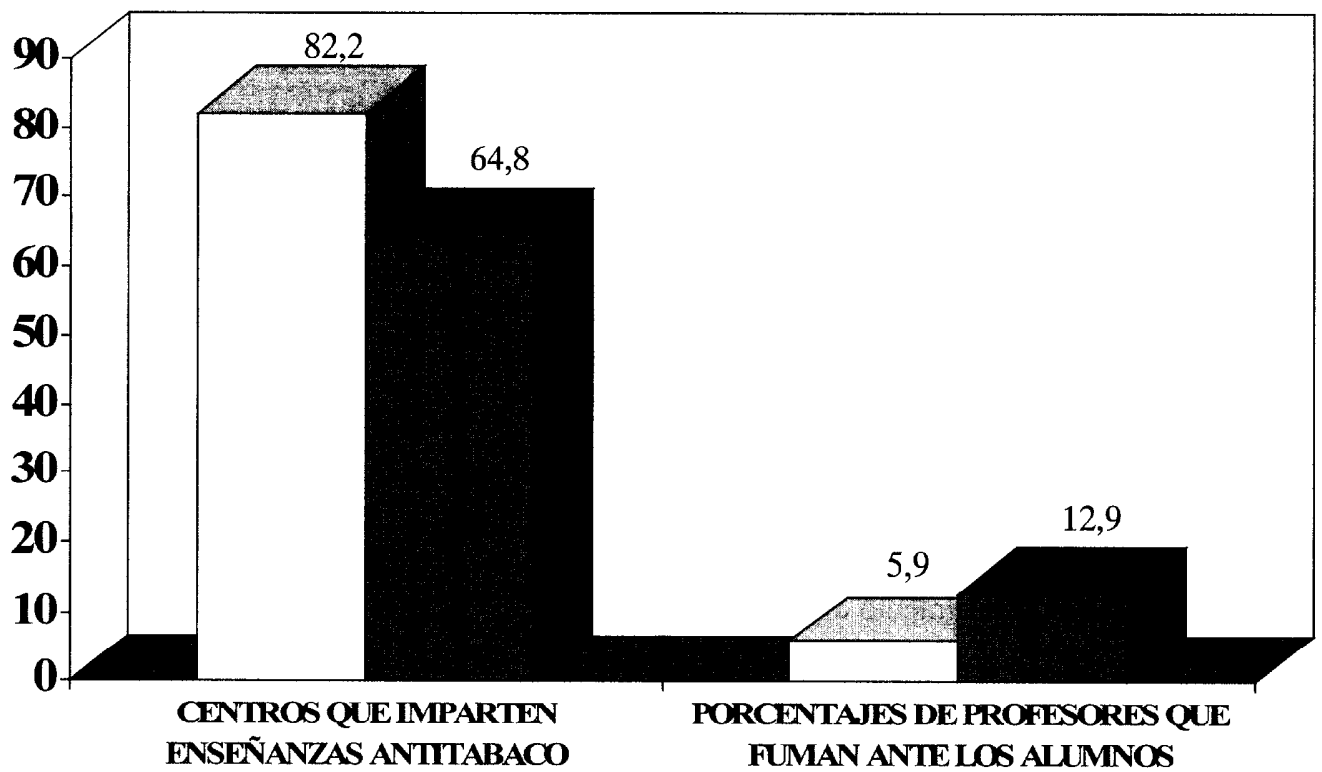

El porcentaje medio estimado de profesores que fuman ante los alumnos es menor en los centros que imparten temas anti-tabaco que en los centros que no los imparten $(6,71 \pm 13,8$ $\%$ frente a $8,07 \pm 16,7 \% ; \mathrm{p}<0,05)$.

El porcentaje medio estimado de alumnos que han probado el tabaco, en los centros donde se imparten temas anti-tabaco, es del $29 \pm 28,1 \%$, y en los centros donde no se imparten del $31 \pm 31 \%(\mathrm{p}<0,001)$. Hay una correlación negativa $(-0,039 ; \mathrm{p}<0,05)$ entre el porcentaje de alumnos que se estima fuman habitualmente y el porcentaje de asignaturas en las que se imparten temas anti-tabaco.

En opinión de los directores escolares, los alumnos de lơs centros que cuentan con carteles anti-tabaco fuman su primer pitillo a una edad media de $12 \pm 2,14$ años, mientras que los alumnos de los centros que no cuentan con este tipo de carteles lo hacen a una edad media de $11,74 \pm 2,26$ años, siendo ésta diferencia significativa $(\mathrm{p}<0,05)$. Existe un coeficiente de correlación de 0,0317 ( $p<0,05$ ) entre la edad a la que se estima que los alumnos fuman su primer pitillo y el porcentaje de asignaturas en las que se imparten enseñanzas anti-tabaco. En la tabla 1 se exponen los principales datos del estudio. 
Tabla 1

Resumen de los principales datos del estudio

\begin{tabular}{|c|c|c|c|}
\hline & $\begin{array}{l}\text { \%o Profesores } \\
\text { Fuman ante } \\
\text { alumnos }\end{array}$ & $\begin{array}{l}\text { \% alumnos } \\
\text { fumadores }\end{array}$ & $\begin{array}{l}\text { Edad media } \\
\text { inicio al } \\
\text { tabaquismo }\end{array}$ \\
\hline Centros que cumplen la Iegislación & $5,56 \pm 12,9$ & $15,33 \pm 20,3$ & $11,55 \pm 3,73$ \\
\hline Centros que no exigen la legislación & $12,9 \pm 20,3$ & $11,18 \pm 16,7$ & $12,41 \pm 2,09$ \\
\hline Centros con carteles antitabaco & $5,80 \pm 12,87$ & $8,94 \pm 13,8$ & $12 \pm 2,14$ \\
\hline Centros sin carteles antitabaco & $7,75 \pm 15,39$ & $17,7 \pm 21,8$ & $11,74 \pm 2,26$ \\
\hline Centros que imparten enseñanzas antitabaco & $6,71 \pm 13,80$ & $14,35 \pm 19,54$ & $11,93 \pm 2,12$ \\
\hline Centros que no imparten enseñanzas antitabaco & $8,07 \pm 16,7$ & $15,05 \pm 20,36$ & $11,84+2,39$ \\
\hline $\begin{array}{l}\text { Coeficiente de correlación entre el porcentaje de } \\
\text { asignaturas en que se imparten enseñanzas antitabaco } \\
\text { y el porcentaje de alumnos fumadores }\end{array}$ & \multicolumn{3}{|c|}{$-0,039(p<0,05)$} \\
\hline $\begin{array}{l}\text { Coeficiente de correlación entre el porcentaje de } \\
\text { asignaturas en que se imparten enseñanzas antitabaco } \\
\text { y la edad a la que los alumnos fuman su primer pitillo }\end{array}$ & \multicolumn{3}{|c|}{$0,031(\mathrm{p}<0,05)$} \\
\hline
\end{tabular}

\section{DISCUSIÓN}

Para valorar el cumplimiento de la legislación anti-tabaco en los centros escolares se han utilizado como criterios el grado de cumplimiento del Real Decreto 192/1988 (que incluye medidas como la prohibición de fumar en los centros docentes, la obligatoriedad de señalizar las áreas donde no se permite fumar y la prohibición de la venta de tabaco en los centros escolares) y los contenidos educativos de EpS y relacionados con la prevención del tabaquismo que los jóvenes reciben. En España, aún nos encontramos en fase de sensibilización acerca del problema y, a pesar de la legislación existente ${ }^{6-11}$, no parecc que la educación anti-tabaco sea una realidad hoy para un gran porcentaje de estudiantes.

La elección de los directores escolares como objetivo diana para interpretar la realidad escolar que tratamos de investigar supone una limitación metodológica ya que los resultados observados son obtenidos a través de la interpretación de la realidad que hacen los propios directores, pero creemos que los resultados descritos por estos se corresponden con la realidad, ya que por ejemplo la prevalencia del consumo de tabaco y las edades de comienzo y consolidación del hábito, que ellos creen existen entre los alumnos, se corresponden bastante fielmente con los datos publicados en estudios realizados entre los propios alumnos y de los que existe numerosa bibliografía ${ }^{15-18}$.

A la hora de interpretar los resultados tenemos que asumir que el hecho de que no hayan contestado la encuesta 4.844 directores puede introducir un sesgo en la información, que puede estar determinado por la suposición de que entre aquellos que sí respondieron podrían ser más los que creen (consciente o inconscientemente) cumplir la legislación, mientras que entre los no respondedores podrían encontrarse principalmente los que no la cumplen.

La mayor parte de los directores de los centros educativos encuestados $(80,9 \%)$ afir- 
man que, por norma, en sus centros exigen el cumplimiento de la legislación oficial anti-tabaco, pero un $19,1 \%$ de ellos reconoce que no se exige, hecho destacable, ya que los centros educativos, junto con los sanitarios, son las instituciones sociales donde los responsables de la administración debieran ser más celosos en el cumplimiento de esta legislación.

Existen carteles recordando la prohibición de fumar en el $64,9 \%$ de centros educativos, lo que significa que más del $35 \%$ no cuentan con carteles y, por tanto, no cumplen la legislación vigente. Estos datos concuerdan con los derivados de otro reciente estudio ${ }^{19}$ realizado en instituciones oficiales de la provincia de Salamanca. No obstante, a pesar de que el $80,9 \%$ de directores declaran exigir el cumplimiento de la legislación oficial en sus centros, menos del $65 \%$ de ellos cuentan con carteles anti-tabaco, lo que da idea del desconocimiento de la legislación por parte de algunos directores. Entre los centros en los que se exige el cumplimiento de la legislación, el porcentaje de centros con carteles es mayor que en los que no la exigen $(68,9 \%$ y $51,5 \%$ respectivamente). Parece interpretarse que una mayor voluntad de hacer cumplir la legislación oficial anti-tabaco se traduce en una mayor presencia de carteles que recuerdan tanto a alumnos como a profesores que en el centro no se permite el uso de tabaco, lo que puede contribuir a disminuir el consumo en el centro por parte de los alumnos. Por tanto, es necesario un esfuerzo adicional para que estos directores con voluntad de hacer cumplir la legislación oficial antitabaco la conozcan en su totalidad y de este modo alcancen los objetivos que se proponen.

En opinión de los directores escolares, los alumnos de los centros que cuentan con carteles anti-tabaco fuman su primer pitillo a una edad media de 12 años, mientras que los alumnos de los centros que no cuentan con este tipo de carteles lo hacen a una edad media de 11,74 años, siendo ésta diferencia significativa. Por tanto, la presencia de carteles que recuerdan la prohibición de fumar en los centros educativos puede contribuir a retrasar la edad a la que los alumnos se inician en el tabaquismo, circunstancia determinante de las características del tabaquismo en la etapa adulta de la vida ${ }^{20,21}$. Por otra parte, el hecho de que la edad media estimada a la que los alumnos fuman su primer pitillo sea ligeramente mayor en los centros en los que se imparten enseñanzas antitabaco se puede explicar porque estas enseñanzas se imparten principalmente en los últimos cursos (en educación secundaria), en los que los alumnos cuentan con mayor edad y, lógicamente, a mayor edad mayor porcentaje de fumadores.

El porcentaje de centros que imparten enseñanzas anti-tabaco es mayor en los que los directores afirman que exigen el cumplimiento de la legislación oficial anti-tabaco que en los que no la exigen $(82,2 \%$ y $64,8 \%$, respectivamente). Además, en los centros en los que supuestamente se afirma exigir el cumplimiento, el porcentaje estimado de profesores que fuman ante los alumnos es del $5,96 \%$, menor que en los centros donde no se exige, en los que este porcentaje es del $12,89 \%$. Estos datos sugieren que la exigencia del cumplimiento de la legislación oficial anti-tabaco en los centros educativos por parte de los directores, es una medida efectiva para que un mayor porcentaje de jóvenes reciban la información necesaria para hacerles comprender las ventajas de no fumar y para que un mayor número de profesores ejerzan el papel ejemplarizante que les corresponde, ya que la actitud de los profesores incide sobre la de los alumnos 22 .

El porcentaje estimado de profesores que fuman ante los alumnos es menor en los centros donde se imparten enseñanzas destinadas a la prevención del tabaquismo. Esto parece indicar que los profesores que fuman ante los alumnos se sienten menos legitimados para impartir enseñanzas que ellos mismos no comparten, lo que priva a muchos alumnos de unos conocimientos que pueden ayudarles a mantenerse alejados del tabaco, ya que las enseñanzas anti-tabaco son útiles, como indica el hecho de que cuando en un centro se impar- 
ten estas enseñanzas es menor el porcentaje de jóvenes que prueban el tabaco y que cuanto mayor es el porcentaje de asignaturas en que se trata este tema, más se retrasa la edad a la que se fuman el primer cigarrillo.

A la luz de estos datos, el cumplimiento de la legislación (presencia de carteles anti- tabaco, ausencia de profesores que fumen ante los alumnos y presencia de enseñanzas anti-tabaco en los currículos de los centros) parece repercutir en un mejor funcionamiento global de los centros, puesto que cada uno de los factores repercute positivamente sobre los otros (figura 4).

Figura 4

Esquema que representa las influencias e interrelaciones derivadas de la exigencia del cumplimiento de la legislación oficial anti-tabaco en los centros educativos, sobre el porcentaje de alumnos fumadores

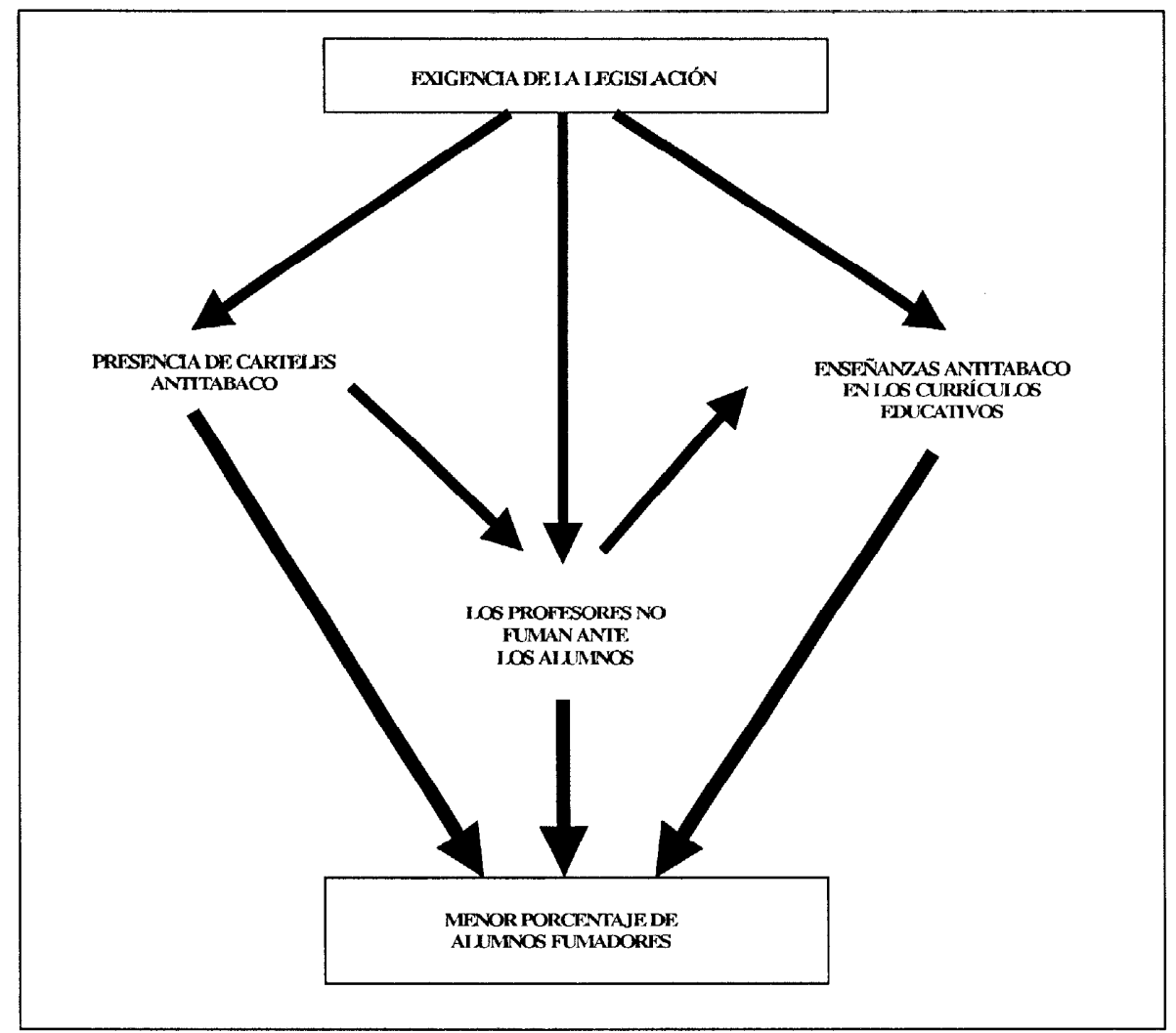

Hay además otra razón que justifica la necesidad de que en los centros educativos se respete la legislación vigente y que ni los alumnos ni los profesores fumen dentro de los recintos escolares. En un reciente estudio llevado a cabo en los Estados Unidos entre adolescentes fumadores ocasionales se demostró que más de la mitad de ellos dejaron de fumar durante el año que duró el estudio ${ }^{23}$ y la mayor parte de los abandonos se produjeron durante periodos vacacionales, lo que indica que para un gran porcentaje de jóvenes fumadores los centros educativos son el medio más favorable, cuando no el único, para fumar. Dicho de otro modo, si no pudieran fumar en los centros escolares, muchos 
jóvenes jamás hubiesen entrado en contacto con el tabaco.

En algunos países de nuestra órbita socio-cultural, como los Estados Unidos, se están propugnando actualmente políticas mucho más rigurosas que las que imperan en España. En el informe final del Comité Asesor sobre Política del Tabaco y Salud Pública de los Estados Unidos, se propugna que en los centros escolares y otras instituciones al servicio de la infancia se adopte e impulse una política de nula tolerancia contra el tabaco, y que esta política se aplique tanto a los menores como a las personas adultas que desempeñan su trabajo en estos centros ${ }^{24}$.

En España se debe avanzar también en esta línea, puesto que es la única forma de lograr que las normas legislativas promulgadas cumplan la misión para la que han sido diseñadas. Es imprescindible que los encargados por parte de la administración de hacer cumplir las normas, en el caso de los centros educativos los directores escolares, vigilen el cumplimiento de esta legislación, puesto que de no ser así de nada sirven los esfuerzos legislativos realizados. Los profesionales sanitarios en general y los médicos en particular (especialmente médicos de familia, internistas y pediatras) debemos colaborar con las escuelas para conseguir que la Educación para la Salud sea una realidad en todas ellas y los niños y jóvenes puedan crecer en un entorno libre de tabaco.

En resumen, el estudio, a pesar de sus limitaciones, derivadas por una parte de la tasa de respuestas (lo que puede inducir un sesgo de información que incluso puede sobreestimar el grado de cumplimiento de la legislación) y por otra del hecho de basarse en las opiniones personales de los directores que han querido colaborar, da idea de que el cumplimiento de la legislación oficial antitabaco en los centros educativos españoles es aún muy deficiente y por ello es preciso un nuevo impulso por parte de las administraciones implicadas, así como motivo de reflexión para los propios profesores, para que el abundante marco normativo vigente surta los efectos buscados. Nuevos estudios diseñados para obtener datos reales del grado de cumplimiento de la legislación en los centros y de los porcentajes de alumnos y profesores fumadores podrían aportar una visión más exacta de la situación real en los centros educativos españoles.

\section{AGRADECIMIENTOS}

A la Sociedad Española de Neumología y Cirugía Torácica (SEPAR), por su apoyo técnico y financiación del estudio. Al Ministerio de Educación y Cultura (MEC), que facilitó el listado informático oficial de los centros educativos del Estado Español. A la Dirección General de Salud Pública y Consumo del Ministerio de Sanidad y Consumo por la presentación pública de los resultados.

\section{BIBLIOGRAFÍA}

1. González de Vega JM, Romero A. Factores inductores en la iniciación al tabaco. En: Aproximación al tabaquismo en España. Madrid: Aula Médica 1997; 39-50.

2. Comisión Interinstitucional Educativa para la Salud de la Provincia de Salamanca. Conducta de fumar en los estudiantes de $7^{\circ}$ y $8^{\circ}$ de E.G.B. Salamanca: Ministerio de Educación y Ciencia; 1996.

3. Ballestín M, Jáuregui $O$, Ribera $S$, Villabí JR. El tabaco en las escuelas primarias. Aten Primaria 1992; 10: 834-6.

4. Barrueco M. Bases legislativas para la prevención del tabaquismo en la escuela. Arch Bronconeumol 1996; 32: 237-41.

5. Real Decreto 192/1988, sobre limitaciones a la venta de tabaco para protección de la salud de la población. BOE n ${ }^{0} 59$ de 9 de Marzo de 1988; 7499-501.

6. Ley Orgánica 1/1990 de Ordenación General del Sistema Educativo. BOE $n^{\circ} 238$ de 4 de Octubre de 1990; 28927-42.

7. Real Decreto 1344/1991 por el que se establece el Currículo de la Educación Primaria. BOE $n^{\circ}$ 220 de 13 de Septiembre de 1991; 30226-8. 
8. Real Decreto $1345 / 1991$ por el que se establece el Currículo de la Enseñanza Secundaria Obligatoria. BOE $\mathrm{n}^{\circ} 220$ de 13 de Septiembre de 1991; 30228-31.

9. Real Decreto $819 / 1993$ por el que se establecen los Reglamentos Orgánicos de las Escuelas de Educación Infantil y de los Colegios de Educación Primaria. BOE n ${ }^{\circ} 146$ de 19 de Junio de 1993; 18891-902.

10. Real Decreto 929/1993 por el que se establecen los Reglamentos Orgánicos de los Institutos de Educación Secundaria. BOE $n^{\circ} 166$ de 13 de Julio de 1993; 20985-21000.

11. Resolución 7/9/94 de la Secretaría de Estado de Educación sobre orientaciones para el desarrollo de la educación en valores en las actividades educativas de los centros docentes. BOE 23 de Septiembre de 1994; 2962-5.

12. Ministerio de Educación y Ciencia. Clasificación de los centros por enseñanza que imparten, por comunidad autónoma. www.estadística@mec.es (consulta octubre 1996).

13. Barrueco M, Hernández-Mezquita MA, Cordovilla R, Vcga MT, Garrido E, Jiménez C. Prevención del tabaquismo en los centros escolares españoles. Arch Bronconeumol 1998; 34: 496-9.

14. Rodríguez J. Métodos de muestreo. Madrid: CIS Educación;1991. p. 322-328.

15. Barrueco M, Vicente M, Garavís JL, García J, Blanco A, Rodríguez MC. Prevención del tabaquismo en la escuela: resultados de un programa realizado durante 3 años. Arch Bronconeumol 1998; 34; 323-328.

16. Díaz E, VillabíJR, Nebot M, Aubá J, Sanz F. El inicio del consumo de tabaco en escolares: estudio transversal y longitudinal de los factores predictivos. Med Clin (Bar) 1998; 110: 334 339.
17. M Barrueco, R Cordovilla, MA Hernández, J,I González, J De Castro, P Rivas,et al. Veracidad en las respuestas de niños, adolescentes y jóvenes a las encuestas sobre el consumo de tabaco realizadas en los centros escolares. Med Clin 1999; 112: 251-254.

18. Alvarez FJ, Vellisco A, Calderón E, Sánchez J, Del Castillo D, Vargas R, et al. Tabaquismo escolar en la provincia de Sevilla. Epidemiología e influencia del entorno personal y social (campaña de prevención del tabaquismo 1998 1999). Arc Bronconeumol 2000; 36: 118-123.

19. Cordovilla R, Barrueco M, González JM, Hernández-Mezquita MA, de Castro J, Gómez F. Cumplimiento de la legislación anti-tabaco en instituciones oficiales. Arch Bronconeumol 1997; 33: 320-4.

20. Center for Disease Control. Reducing the health consequences of smoking: 25 years of progress. A report of The Surgeon General. Washington DC: US Department of Health and Human Services. Public Health Service, CDC; 1989; DHHS publication $\mathrm{n}^{\circ}$. 89-8411.

21. Taioli E, Wynder E. Effect of the age at which smoking begins on frecuency of smoking in adulthood. N Engl J Med 1991; 325: 968-9.

22. Barrueco M, Hernández-Mezquita MA, Jiménez C Cordovilla R, Flores S, Vega MT, Garrido $E$. Influence of the teachers smoking habits on the educative anti-tobacco contents that they impart. Am Rev Respir Dis 1999; 159: 487.

23. Hines D, Nollen N, Fretz AC. One-year follow up of college students occasional smokers. Tobacco Control 1996; 5: 231-2.

24. Final Report of the Advisory Committee on Tobacco Policy and Public Health. Tobacco Control 1997; 6: 254-61. 
Anexo 1

Principales preguntas incluidas en la encuesta remitida a los directores de centros educativos

- ¿Qué porcentaje de alumnos de su centro cree que han probado el tabaco?

- ¿Qué porcentaje de alumnos de su centro cree que fuman habitualmente?

- ¿Qué porcentaje de profesores de su centro cree que son fumadores?

- ¿Qué porcentaje de profesores de su centro cree que fuman ante los alumnos?

- ¿A qué edad cree que los alumnos de su centro prueban su primer pitillo? (En años)

- ¿A qué edad cree que los alumnos de su centro pasan a ser fumadores habituales? (En años)

- En su centro, por norma, ¿Se exige el cumplimiento de la legislación oficial anti-tabaco?

- ¿Existen en su centro carteles advirtiendo de la prohibición de fumar?

- ¿En su centro, se trata el tema del tabaco dentro de la Educación para la Salud?

- ¿En qué porcentaje de asignaturas se trata el tema del tabaco en su centro? 